Original paper

\title{
Efficacy and direct costs of chronic hepatitis C treatment with first generation NS3/4A protease inhibitors in a real life population
}

Anna Piekarska', Ewa Koślińska-Berkan' ${ }^{1}$ Kamila Wójcik' ${ }^{1}$ Anna Skubała', Maciej Jabłkowski², Zbigniew Deroń³, Aleksandra Berkan-Kawińska'

'Department of Infectious Diseases and Hepatology, Medical University of Lodz, Poland

2Department of Infectious and Liver Diseases, Medical University of Lodz, Poland

${ }^{3}$ Observation - Infectious and Hepatology Department, Bieganski Voivodship Specialist Hospital in Lodz, Poland

\begin{abstract}
Introduction: Recent years have brought a significant advance in chronic hepatitis $\mathrm{C}(\mathrm{CHC})$ treatment that includes development of direct acting antivirals (DAA). Two of them, boceprevir (BOC) and telaprevir (TVR), were first approved for treatment of patients infected with $\mathrm{CHC}$ genotype 1 in combination with pegylated interferon (P) and ribavirin (R). Our aim was to evaluate the efficacy and direct costs of BOC/PR and TVR/PR in a real life population.

Material and methods: The study included adult patients qualified for the CHC Therapeutic Programme treated with TVR/PR or BOC/PR. Treatment was continued for 24 or 48 weeks. Sustained virological response, treatment discontinuation due to adverse events and lack of virological response rates were compared.

Results: A total of 243 adult patients with CHC were included. TVR/PR and BOC/PR were administered in respectively 122 and 121 patients. Thirty-two patients (13\%) were treatment-naïve, whereas liver cirrhosis/advanced fibrosis was observed in 138 patients (56.7\%). Overall, $43.6 \%$ of patients achieved a sustained virologic response (SVR). In the BOC/PR group the SVR rate was significantly lower than in the TVR/PR group (33.1\% vs. 54.1\%; $p=0.00094)$. Lack of response to therapy was observed in $41.3 \%$ and $12.3 \%$ of patients receiving BOC and TVR, respectively $(p<0.00001)$. The direct cost of achieving SVR in one patient was 285450 PLN with BOC and 185757 PLN with TVR.

Conclusions: The very low treatment efficacy may be the result of inclusion criteria that allowed treatment of patients with advanced liver fibrosis/liver cirrhosis or previous treatment failure. Telaprevir seems to be significantly more potent against hepatitis C virus, with similar safety and tolerance.
\end{abstract}

Key words: hepatitis C, boceprevir, first-generation protease inhibitors, sustained virologic response, telaprevir.

\section{Address for correspondence}

Aleksandra Berkan-Kawińska, Department of Infectious Diseases and Hepatology, Medical University of Lodz, 1/5 Kniaziewicza St., 91-347 Lodz, Poland, phone: +48 661191 396, e-mail: aleksandra.berkan@gmail.com

\section{Introduction}

Chronic hepatitis $\mathrm{C}(\mathrm{CHC})$ persists as a major epidemiological issue in Poland. According to Polish Expert Group estimates approximately $0.6 \%$ of the whole Polish population may be chronically infected with hepatitis $\mathrm{C}$ virus (HCV) [1]. Recent years have brought a significant advance in $\mathrm{CHC}$ treatment that includes development of direct acting antivirals (DAA). First generation DAA - NS3/4A protease inhibitors - were included in the National Therapeutic Programme for Hepatitis C in 2013. Two of them (boceprevir [BOC] and telaprevir [TVR]) were approved by the European Medicines Agency and reimbursed by the Polish Ministry of Health for patients infected with HCV genotype 1 in combination with interferon and ribavirin. Results of 
clinical trials (boceprevir/pegylated interferon/ribavirin $[\mathrm{BOC} / \mathrm{PR}]$ and telaprevir/pegylated interferon/ribavirin $[\mathrm{TVR} / \mathrm{PR}]$ ) were encouraging - the new agents seemed to exhibit very strong antiviral activity that exceeded the efficacy of dual therapy based on pegylated interferon and ribavirin (PR), with the sustained viral response (SVR) ranging from 66 to $75 \%$ [2-4].

Nevertheless, the high antiviral potential of BOC and TVR reported in clinical trials was observed mostly in naïve patients (only if IL-28B TT polymorphism was present), as well as in patients with a low fibrosis stage (F0-F2 on the METAVIR scale). However, according to the National Therapeutic Programme for Hepatitis C, NS3/4A protease inhibitor-based therapy was available only in treatment-experienced patients with a fibrosis stage of at least F2. Such eligibility criteria limited treatment expenses nationally, but resulted in decreased cost-effectiveness and augmented risk of serious adverse events observed especially in patients with liver cirrhosis [5].

Our aim was to evaluate the efficacy of first generation protease inhibitors in patients with $\mathrm{CHC}$ and to compare the direct costs of $\mathrm{BOC} / \mathrm{PR}$ and TVR/PR therapies in a real life population.

\section{Material and methods}

\section{Inclusion criteria}

The following analysis is a retrospective evaluation of efficacy and direct costs of BOC/TVR-based therapies in patients qualified for the Hepatitis C Therapeutic Programme valid at the moment of study (currently replaced by an interferon-free regimen) within routine clinical practice. The study included adult patients treated with $\mathrm{TVR} / \mathrm{PR}$ or $\mathrm{BOC} / \mathrm{PR}$ who completed a 6-month follow-up after treatment.

Inclusion criteria were as follows:

1) chronic hepatitis $C$ infection (positive serum antiHCV antibodies, detection of HCV RNA in serum/ liver),

2) HCV genotype 1 infection,

3) age $\geq 18$ years,

4) treatment-experienced patients (treatment-naïve only if IL-28B TT polymorphism present),

5) liver fibrosis confirmed with liver biopsy or transient elastography in patients with contraindications to liver biopsy: $\geq F 2$ in treatment-naïve or $\geq F 1$ in treatment-experienced (PR therapy) patients.

The exclusion criteria were as follows:

1) patients with clinical manifestations of liver failure (liver cirrhosis of classes B and C according to the Child-Pugh classification),

2) contraindications to interferon therapy,
3) severe concomitant diseases (severe cardiovascular disease, poorly controlled diabetes, autoimmune diseases, hyperthyroidism, retinopathy, drug-resistant epilepsy, severe psychosis, active neoplastic disease),

4) active alcohol and/or psychoactive drug abuse,

5) pregnancy and lactation,

6) HIV/HBV coinfection,

7) positive history of liver transplant.

\section{Medication}

Boceprevir was administered in a dose of $800 \mathrm{mg}$ three times daily whereas TVR was administered in a dose of $1125 \mathrm{mg}$ twice a day, both in combination with pegylated interferon alfa-2a or alfa- $2 b$ and ribavirin. The standard dose of pegylated interferon alfa-2a was $180 \mu \mathrm{g}$ per week and the standard dose of pegylated interferon alfa- $2 \mathrm{~b}$ was $1.5 \mu \mathrm{g}$ per kilogram per week. The baseline ribavirin dose was $1200 \mathrm{mg}$ in patients with body weight $>75 \mathrm{~kg}$ and $1000 \mathrm{mg}$ in patients with body weight $<75 \mathrm{~kg}$. Treatment was continued for 24 or 48 weeks. Length of the therapy and indications to treatment discontinuation due to lack of virological response were determined in accordance with the Summaries of Product Characteristics for TVR and BOC.

\section{Methods}

Demographic data were collected retrospectively using a database that included all patients treated with BOC or TVR. Anti-HCV antibodies were tested by a thirdgeneration enzyme-linked immunoassay (ELISA). HCV RNA was assessed by the reverse transcription PCR (RT-PCR) method with COBAS AMPLICOR HCV v. 2.0 (Roche Diagnostic Inc.). The lower limit of detection was $15 \mathrm{IU} / \mathrm{ml}$. HCV genotype was determined by a hybridization method (InnoLipa HCV, Innogenetics). Quantitative serum HCV RNA was assessed in all patients at baseline, at weeks 4, 12, 24 and at the end of treatment. Early virological response (EVR) was defined as undetectable HCV RNA after 12 weeks of treatment. Lack of response to treatment was diagnosed when the serum HCV RNA level remained above the limit of detection throughout the therapy. The efficacy end point was sustained virological response (SVR), defined as undetectable HCV RNA at 24 weeks after the end of treatment.

Liver biopsy was performed in 52 patients and a histological evaluation was carried out according to the METAVIR classification system. Transient elastography was performed in patients according to the manufacturer's instructions (FibroScan, Echosens). 
The results were expressed in kilopascals $(\mathrm{kPa})$ applying the following cut-off values to determine the degree of fibrosis [6]: $<7.5 \mathrm{kPa}-$ mild or no fibrosis (F0-F1); $7.5 \mathrm{kPa}$ and $<9.5 \mathrm{kPa}-$ significant fibrosis (F2); $\geq 9.5 \mathrm{kPa}$ and $<12.5 \mathrm{kPa}$ - severe fibrosis (F3); $\geq 12.5 \mathrm{kPa}$ - liver cirrhosis (F4).

The variables were compared between patients with SVR and those without as follows: type of treatment (TVR vs. BOC), stage of fibrosis and history of interferon treatment. In patients without SVR we further compared the rates of treatment discontinuation due to adverse events or lack of virological response.

The cost of medications was estimated based on their invoice prices financed by the National Health Fund. The amount of medications used by each patient until treatment end/discontinuation was calculated. The costs of other services provided within the National Therapeutic Programme for Hepatitis C were not included in the analysis.

\section{Statistical analysis}

Quantitative data were presented as mean and median. Qualitative data were characterised by number and percentage distribution and assessed by Pearson's $\chi^{2}$ test. Statistical significance was accepted as $p<0.05$.

\section{Results}

\section{Safety and efficacy}

A total of 243 adult patients with chronic hepatitis $C$ genotype 1 (138 men and 105 women aged 22-76, mean age: 49.2 years) who received antiviral treatment between July 2013 and June 2016 in Biegański Hospital in Lodz according to the criteria of the $\mathrm{Na}$ tional Therapeutic Programme for Hepatitis C were included in the study. Patients finished antiviral treatment and 24 weeks of follow-up. TVR/PR and BOC/ $\mathrm{PR}$ were administered in respectively 122 and 121 patients.

Thirty-two patients ( $13 \%$ of the whole study population) were treatment-naïve, whereas 211 patients (87\%) experienced failure of previous pegylated interferon and ribavirin therapy. Rates of treatment-experienced patients in BOC and TVR groups were respectively 90.1\% and $83.6 \%$. Liver cirrhosis or advanced liver fibrosis (F3/F4) was observed in 138 patients (56.7\%), while mild liver fibrosis (F1/F2) was observed in 105 patients $(43.3 \%)$. In BOC-treated and TVR-treated groups liver cirrhosis or advanced fibrosis was observed in respectively $60.3 \%$ and $53.3 \%$ of patients.

The differences in age, sex, history of treatment and fibrosis observed between the two groups were statisti- cally insignificant. Baseline characteristics of the study population are presented in Table 1.

Overall, 43.6\% (106/243) of patients achieved an $\mathrm{SVR}$. In the BOC/PR group the SVR rate was significantly lower than in the TVR/PR group $(33.1 \%$ [40 patients] vs. 54.1\% [66 patients]; $p=0.00094$ ). A statistically significant difference in SVR rates was also observed in a subgroup of patients with advanced liver fibrosis, who responded significantly better to TVR than to BOC $(52.3 \%$ vs. $27.4 \%$, respectively; $p=0.00276$ ).

Similarly, SVR rates among treatment-experienced patients were significantly higher in the TVR/ $\mathrm{PR}$ group $-53.9 \%$ vs. $34.9 \%$ in the $\mathrm{BOC} / \mathrm{PR}$ group

Table 1. Baseline characteristics of the study population

\begin{tabular}{|c|c|c|c|}
\hline Factor & Telaprevir & Boceprevir & $p$ \\
\hline Number of patients & 122 & 121 & \\
\hline \multicolumn{4}{|l|}{ Age } \\
\hline Mean & 47.4 & 51.0 & \multirow{3}{*}{0.059998} \\
\hline Median & 51 & 55 & \\
\hline Range & $22-74$ & $22-76$ & \\
\hline \multicolumn{4}{|l|}{ Sex, $n(\%)$} \\
\hline Women & $49(40.2 \%)$ & $56(46.3 \%)$ & \multirow{2}{*}{0.33582} \\
\hline Men & $73(59.8 \%)$ & $65(53.7 \%)$ & \\
\hline \multicolumn{4}{|c|}{ History of treatment, $n(\%)$} \\
\hline Naive & $20(16.4 \%)$ & $12(9.9 \%)$ & \multirow{2}{*}{0.13552} \\
\hline Experienced & $102(83.6 \%)$ & $109(90.1 \%)$ & \\
\hline \multicolumn{4}{|l|}{ Liver fibrosis, $n(\%)$} \\
\hline Low (F0-2) & $57(46.7 \%)$ & $48(39.7 \%)$ & \multirow{2}{*}{0.26719} \\
\hline Advanced (F3-4) & $65(53.3 \%)$ & $73(60.3 \%)$ & \\
\hline
\end{tabular}

Table 2. Treatment outcomes in the study groups

\begin{tabular}{|c|c|c|c|}
\hline Factor & Telaprevir & Boceprevir & $p$ \\
\hline \multicolumn{4}{|l|}{ SVR rates, $n(\%)$} \\
\hline Overall & $66(54.1 \%)$ & $40(33.1 \%)$ & 0.00094 \\
\hline \multicolumn{4}{|l|}{ History of treatment } \\
\hline Naive & $11(55 \%)$ & $2(16.7 \%)$ & 0.03256 \\
\hline Experienced & $55(53.9 \%)$ & 38 (34.9\%) & 0.00533 \\
\hline \multicolumn{4}{|l|}{ Liver fibrosis } \\
\hline Low (F0-2) & $32(56.1 \%)$ & $20(41.7 \%)$ & 0.13948 \\
\hline Advanced (F3-4) & $34(52.3 \%)$ & $20(27.4 \%)$ & 0.00276 \\
\hline \multicolumn{4}{|c|}{ Treatment discontinuation, $n(\%)$} \\
\hline Adverse events & $20(16.4 \%)$ & $25(20.7 \%)$ & 0.39182 \\
\hline Lack of response & $15(12.3 \%)$ & $50(41.3 \%)$ & $<0.00001$ \\
\hline
\end{tabular}


$(p=0.00533)$. Lack of response to therapy was observed in $50(41.3 \%)$ patients receiving BOC and in $15(12.3 \%)$ patients receiving TVR $(p<0.00001)$.

Treatment discontinuation due to adverse events was more prevalent in the BOC/PR group $(20.6 \%$ vs. $16.4 \%)$, but the difference was not statistically significant $(p>0.05)$.

Rates of treatment responses are summarized in Table 2.

\section{Direct costs of achieving sustained virologic response}

The overall cost of BOC and TVR treatment was calculated by adding the costs of all initiated therapies. Cost of a single therapy was assessed based on its length (after adjustment to discontinuation due to adverse events or lack of response). Direct cost of achieving SVR in one patient was obtained after dividing the overall cost of treatment by the number of patients receiving SVR. The overall expenses for the treatment in 121 patients treated with BOC/PR totalled 11418000 Polish zloty (PLN), while the direct cost of achieving an SVR in one patient was as high as PLN 285 450. In the TVR/PR group the cost of the medications amounted to PLN 12260000 and achieving one SVR required PLN 185 757. Consequently, the cost of achieving an SVR in one patient with TVR therapy equalled $65 \%$ of the expenses for BOC.

\section{Discussion}

In our study an SVR was achieved in 106 out of 243 (43.6\%) patients with chronic hepatitis C (genotype 1) receiving therapy based on pegylated interferon, ribavirin and BOC or TVR. The sustained virologic response rate of around $43 \%$ is much below the results reported in registration studies of both NS3/4A protease inhibitors [2-4]. It should be noted that the efficacy of BOC/TVR-based treatment is even worse than the efficacy of pegylated interferon-ribavirin therapy of 50-55\% reported in other Polish studies $[7,8]$. This difference may be the result of the high proportion of cirrhotic patients $(138 / 243,56.7 \%)$ and treatmentexperienced patients $(211 / 243,87 \%)$ included in the study population. Such patients are at the highest risk of developing liver failure and hepatocellular carcinoma and, for that reason, had their treatment initiated as a priority. Moreover, inclusion of patients who did not respond to previous therapies was a consequence of the National Therapeutic Programme for Hepatitis C eligibility criteria, according to which NS3/4A protease inhibitor-based therapy was dedicated mainly to treat- ment-experienced patients. Other restrictions such as inclusion of patients with severe fibrosis or patients with interleukin 28B TT subtype (a negative predictor of virologic response) may have also contributed to the low SVR rates and exceptionally high costs of the treatment. Similarly poor efficacy in study groups with high rates of treatment-experienced patients and patients with liver cirrhosis was reported by Sethi et al. [9] (48.5\%) and Bichoupan et al. [10] (44\%).

It is worth noting that the SVR rate in patients treated with TVR was significantly higher than in patients treated with BOC ( $54.1 \%$ vs. $33.1 \%$; $p=0.00094)$. The two groups did not differ significantly in terms of age, sex, liver fibrosis and history of previous treatment. Although both drugs are members of the same class of antiviral agents - known as first generation NS3/4A protease inhibitors - their efficacy in a real life population varies and reflects the actual potential of each active substance. This observation was first reported in the Cupic study [5], where SVR rates in treatment-experienced, cirrhotic patients treated with BOC were lower than in patients treated with TVR. In a study by Salmeron et al. the rate of SVR by intention-to-treat analysis was also greater in patients treated with TVR (65\%) than in patients treated with BOC (52\%, $p<0.0001)$; however, in modified intention to treat analysis the observation was not statistically significant [11]. Differences in SVR rates between TVR and BOC have also been noted by other authors: $53 \%$ vs. $40 \%$ [12] and $56 \%$ vs. $53 \%$, respectively [13]. None of the studies published reported an advantage of BOC.

Similar rates of TVR efficacy were observed in another Polish "real life" study investigating safety and efficacy of triple therapy with TVR in treatment-experienced patients with advanced liver fibrosis [14]. The overall SVR24 rate in this analysis was 56\% and was lower in cirrhotic patients than in patients with bridging fibrosis. These observations are consistent with the results of our study.

Rates of lack of response seem to confirm significant differences in antiviral potential of the two drugs. Non-responders to BOC or TVR constituted $41.3 \%$ and $12.3 \%$ of the total, respectively ( $p=0.0001$ ). This clear difference may be suggestive of low ability of BOC to reduce viral load, even when administered after a 4-week lead-in of pegylated interferon and ribavirin.

At the time of our study it was believed that TVR posed a higher risk of severe adverse events. Moreover, tolerance of TVR-based therapies was also expected to be worse. Our retrospective analysis of both NS3/4A protease inhibitors in comparable groups of patients proves that rates of treatment discontinuation due to adverse events, although not significantly different, 
were even higher in patients treated with BOC $(20.7 \%$ vs. $16.4 \%, p>0.05)$. Another Polish study yielded a similar rate of serious adverse events caused by TVR (15\%) [14].

The different SVR rates for TVR and BOC observed in our study resulted in diversification of direct costs of each drug. Achieving an SVR in one patient cost PLN 285450 if treated with BOC and PLN 185757 if treated with TVR. This observation is in accordance with results obtained by other researchers, who also reported that treatment with TVR is less expensive and therefore more effective than treatment with BOC $[15,16]$. Only Giménez-Manzorro et al. did not observe any significant difference in costs of the two drugs [17].

Therefore, the very high costs of antiviral agents used in the years 2013-2015 seem not to entail satisfactory therapeutic effects. However, in more detailed analyses that included the economic setting of European countries, the USA or Singapore, several authors proved that therapies based on first generation protease inhibitors were still cost-effective [10, 15, 16, 18, 19].

\section{Conclusions}

The results of our study prove that treatment with first generation NS3/4A protease inhibitors administered within the National Therapeutic Programme for Hepatitis yielded lower SVR rates and significantly higher costs than reported in their registration trials. The very low treatment efficacy may be the result of inclusion criteria that allowed treatment of patients with negative prognostic factors, such as advanced liver fibrosis/liver cirrhosis or previous treatment failure. Out of the two first generation NS3/4A protease inhibitors, TVR seems to be significantly more potent against hepatitis $\mathrm{C}$ virus, with similar safety and tolerance.

\section{Disclosure}

Authors report no conflict of interest.

\section{References}

1. Halota W, Flisiak R, Boroń-Kaczmarska A, et al. Recommendations for the treatment of hepatitis $\mathrm{C}$ issued by the Polish Group of HCV Experts - 2016. Clin Exp Hepatol 2016; 2: 27-33.

2. McHutchison JG, Everson GT, Gordon SC, et al. Telaprevir with peginterferon and ribavirin for chronic HCV genotype 1 infection. N Engl J Med 2009; 360: 1827-1838.

3. Hézode C, Forestier N, Dusheiko G, et al. Telaprevir and peginterferon with or without ribavirin for chronic HCV infection. N Engl J Med 2009; 360: 1839-1850.

4. Kwo PY, Lawitz EJ, McCone J, et al. Efficacy of boceprevir, an NS3 protease inhibitor, in combination with peginterferon alfa- $2 \mathrm{~b}$ and ribavirin in treatment-naive patients with genotype 1 hepatitis $C$ infection (SPRINT-1): an open-label, randomised, multicentre phase 2 trial. Lancet 2010; 376: 705-716.

5. Hézode C, Fontaine H, Dorival C, et al. Effectiveness of telaprevir or boceprevir in treatment-experienced patients with HCV genotype 1 infection and cirrhosis. Gastroenterology 2014; 147: 132-142.e4.

6. Castera L, Vergniol J, Foucher J, et al. Prospective comparison of transient elastography, Fibrotest, APRI, and liver biopsy for the assessment of fibrosis in chronic hepatitis C. Gastroenterology 2005; 128: 343-350.

7. Juszczyk J, Baka-Cwierz B, Beniowski M, et al. Pegylated interferon-alfa $2 a$ with ribavirin in chronic viral hepatitis $\mathrm{C}$ (final report). Przegl Epidemiol 2005; 59: 651-660.

8. Piekarska A, Zboińska J, Wójcik K, et al. The efficacy of various combinations of antiviral drugs in retherapy of chronic hepatitis C patients. Exp Clin Hepatol 2006; 2: 41-45.

9. Sethi N, Tapper EB, Vong A, et al. Direct costs of first-generation protease inhibitors for the treatment of genotype 1 chronic hepatitis C viral infection. J Viral Hepat 2015; 22: 974-976.

10. Bichoupan K, Martel-Laferriere V, Sachs D, et al. Costs of telaprevir-based triple therapy for hepatitis C: $\$ 189,000$ per sustained virological response. Hepatology 2014; 60: 1187-1195.

11. Salmerón J, Vinaixa C, Berenguer R, et al. Effectiveness and safety of first-generation protease inhibitors in clinical practice: Hepatitis C virus patients with advanced fibrosis. World J Gastroenterol 2015; 21: 9163-9174.

12. Vo KP, Vutien P, Akiyama MJ, et al. Poor Sustained Virological Response in a Multicenter Real-Life Cohort of Chronic Hepatitis C Patients Treated with Pegylated Interferon and Ribavirin plus Telaprevir or Boceprevir. Dig Dis Sci 2015; 60: 1045-1051.

13. Price JC, Murphy RC, Shvachko VA, et al. Effectiveness of telaprevir and boceprevir triple therapy for patients with hepatitis $\mathrm{C}$ virus infection in a large integrated care setting. Dig Dis Sci 2014; 59: 3043-3052.

14. Janczewska E, Flisiak R, Zarebska-Michaluk D, et al. Effect of Peginterferon or Ribavirin Dosing on Efficacy of Therapy With Telaprevir in Treatment-Experienced Patients With Chronic Hepatitis C and Advanced Liver Fibrosis: A Multicenter Cohort Study. Medicine (Baltimore) 2015; 94: e1411.

15. Camma C, Petta S, Cabibbo G, et al. Cost-effectiveness of boceprevir or telaprevir for previously treated patients with genotype 1 chronic hepatitis C. J Hepatol 2013; 59: 658-666.

16. Cortesi PA, Ciaccio A, Rota M, et al. Management of treatment-naïve chronic hepatitis $\mathrm{C}$ genotype 1 patients: a cost-effectiveness analysis of treatment options. J Viral Hepat 2015; 22: 175-183.

17. Giménez-Manzorro Á, García-González X, Rodríguez-González CG, et al. Cost-effectiveness and safety of telaprevir and boceprevir for chronic hepatitis $\mathrm{C}$ in real-world clinical practice. Gastroenterol Hepatol 2015; 38: 575-582.

18. Dan YY, Ferrante SA, Elbasha EH, et al. Cost-effectiveness of boceprevir co-administration versus pegylated interferon- $\alpha 2 b$ and ribavirin only for patients with hepatitis $\mathrm{C}$ genotype 1 in Singapore. Antivir Ther 2015; 20: 209-216.

19. Athanasakis K, Ferrante SA, Kyriopoulos II, et al. Boceprevir for Chronic Genotype 1 Hepatitis C Virus in the Current Health Care Setting in Greece: A Cost-effectiveness Analysis. Clin Ther 2015; 37: 1529-1540. 